\title{
Should statin guidelines consider patient preferences? Eliciting preferences of benefit and harm outcomes of statins for primary prevention of cardiovascular disease in the sub-Saharan African and European contexts
}

Henock G. Yebyo ${ }^{1,2}$, Hélène E. Aschmann ${ }^{1}$, Tsung Yu ${ }^{3}$ and Milo A. Puhan ${ }^{1 *}$ (D)

\begin{abstract}
Background: Patient preferences are key parameters to evaluate benefit-harm balance of statins for primary prevention but they are not readily available to guideline developers and decision makers. Our study aimed to elicit patient preferences for benefit and harm outcomes related to use of statins for primary cardiovascular disease prevention and to examine how the preferences differ across economically and socio-culturally different environments.

Methods: We conducted preference-eliciting surveys using best-worst scaling designed with a balanced incompleteblock design (BIBD) on 13 statins-related outcomes on 220 people in Ethiopia and Switzerland. The participants made tradeoff decisions and selected the most and least worrisome outcomes concurrently from each scenario generated using the BIBD. The design yielded 34,320 implied paired-comparisons and 2860 paired-responses as unit of analysis for eliciting the preferences that were analyzed using a conditional-logit model on a relative scale and surface under the cumulative ranking curve from multivariate random-effects meta-analysis model on a scale of 0 to 1.

Results: There was high internal consistency of responses and minimal amount of measurement error in both surveys. Severe stroke was the most worrisome outcome with a ceiling preference of 1 (on 0 to 1 scale) followed by severe myocardial infarction, 0.913 (95\% Cl, 0.889-0.943), and cancer, 0.846 (0.829-0.855); while treatment discontinuation, 0.090 $(0.023-0.123)$, and nausea/headache, $0.060(0.034-0.094)$ were the least worrisome outcomes. Preferences were similar between Ethiopia and Switzerland with overlapping uncertainty intervals and concordance correlation of 0.97 (0.90-0.99). Conclusions: Our study provides much needed empirical evidence on preferences that help clinical guidelines consider for weighing the benefit and harm outcomes when recommending for or against statins for primary prevention of cardiovascular disease. The preferences are consistent across the disparate settings; however, we recommend inclusion of more countries in future studies to ensure the generalizability of the preferences to all environments.
\end{abstract}

Keywords: Preferences, Statins, Benefit harm outcomes, Primary prevention, Cardiovascular disease, Clinical guidelines

\footnotetext{
* Correspondence: miloalan.puhan@uzh.ch

1 Department of Epidemiology, Biostatistics \& Prevention Institute, University

of Zurich, Hirschengraben 84, CH-8001 Zurich, Switzerland

Full list of author information is available at the end of the article
}

(c) The Author(s). 2018 Open Access This article is distributed under the terms of the Creative Commons Attribution 4.0 International License (http://creativecommons.org/licenses/by/4.0/), which permits unrestricted use, distribution, and reproduction in any medium, provided you give appropriate credit to the original author(s) and the source, provide a link to the Creative Commons license, and indicate if changes were made. The Creative Commons Public Domain Dedication waiver (http://creativecommons.org/publicdomain/zero/1.0/) applies to the data made available in this article, unless otherwise stated. 


\section{Background}

Statins are among the most widely used drugs for primary and secondary prevention of cardiovascular disease (CVD) [1]. Current clinical guidelines have progressively lowered the threshold to initiate statins for primary prevention to 7.5-10\% 10-year risk for CVD [2]. As a result, millions of healthy people may be indicated for statins as life-long medication. According to the American College of Cardiology/American Heart Association guideline, for example, $24 \%$ of the U.S. population is ought to use statins [3]. Until recently, the use of statins has been limited to affluent regions despite the fact that threefourths of global CVD deaths occur in low- and middleincome countries $[4,5]$. However, the recently improved availability of less expensive generic statins has made them widely accessible to the developing world as well [6].

In spite of their wider use, it is remarkable that existing clinical guidelines do not explicitly assess whether the harms related to statins are trivial enough to be ignored or offset their benefits [7]. Randomized controlled trials (RCTs) have shown statins' efficacy in reducing risk of new CVD but also reported harmful effects, such as type 2 diabetes, cancer, and hepatic and renal dysfunctions $[8,9]$. Clinical guidelines heavily rely on relative effects of statins, which alone cannot justify their use [1, 10]. It is essential to take into account absolute risks of benefit and harm outcomes related to the drugs calculated from the number of prevented outcomes or caused harmful events in people taking statins versus people not taking statins [11]. Moreover, considering social context and people's preferences is fundamental to move forward to informed medical practices [12]. Some outcomes are more important than others according to the preferences of individuals and thus need to be weighted accordingly when estimating the benefit-harm balance of statins and framing recommendations. Advanced methods that analyze all key parameters-relative effect size, baseline risk and preferences-to quantitatively assess benefit-harm balance have become available that go far beyond the number needed to treat and number needed to harm $[11,13]$.

While evidence on treatment effects and baseline risks is fairly available, evidence on the preferences, which is a defining parameter to perform benefit-harm analysis, is mostly lacking [14]. Some studies, including the European and Global Burden of Diseases (GBD), aiming at measuring disability-adjusted life-years (DALYs), estimated disability weights-similar to preference weightsfor a wide array of diseases $[15,16]$. These estimates, however, lack specificity with respect to certain clinical decision contexts, as in the case of prescribing statins for primary prevention of CVD. Furthermore, preferences may vary across populations living in different health systems and social-cultural environments, which may have a substantial impact on the benefit-harm balance of statins and guideline developments [15, 17]. In light of these, we aimed to elicit preferences for benefit and harm outcomes related to the use of statins with emphasis on primary prevention of CVD, and compare preferences between two populations in Sub-Saharan Africa and Europe who live in greatly differing settings in terms of health systems and socio-cultural perspectives.

\section{Methods}

\section{Study design and participants}

We conducted a preference-eliciting survey in Mekelle, Ethiopia, and Zurich, Switzerland from September to December 2016. We considered these environments that differ in terms of health system, socio-cultural and economical statuses, and hence to serve well for comparison of the preferences. Participants had to be 40 years or older and without a history of CVD events. We did not apply any other in- or exclusion criteria.

We obtained the preferences on the predetermined salient benefit and harm outcomes related to statins in the survey using face-to-face interviews. In Ethiopia, the survey was conducted on a home-to-home basis whose respondents were sampled using a computer-assisted random number generator from an existing sampling frame designed for a WHO-stepwise survey. The randomly selected households were visited at convenient times and revisited up to three times when the eligible respondents were not available. The respondents were provided a mobile card for their time that was worth CHF 2.50, an amount equivalent to the market value of the compensation provided in Switzerland. In Zurich, we invited participants from people who visited the travel clinic of the University of Zurich. The walk-in clinic renders service of pre-travel advice on preventive measures including vaccinations, to around 20,000 travelers each year. We considered the setting of the travel clinic to provide a good source for recruitment of participants since the demographic profile of visitors to the clinic is similar to that of the Swiss general population [18]. We thus presumed that these individuals represent the general population quite well. The study participants were interviewed face-to-face. They were compensated with a free consultation at the travel clinic that was worth CHF 50.

\section{Questionnaire design and procedure}

We designed our study to yield preference weights for a higher-level decision-i.e., to help clinical guideline developers to assess benefit-harm balance of statins, for which knowledge about preference is essential. Since guideline developers have to rely on the published evidence on the effectiveness of statins, we decided to include 13 statinassociated benefit ad harm outcomes in the survey that 
were reported in RCTs/meta-analyses $[8,9,19,20]$. The starting point for measuring preferences is a clear definition of the outcomes to be measured because the different outcomes have wide spectrum of manifestation. For example, the statin-associated muscle adverse effects have ranges of presentation from myalgia to rhabdomyolysis [21]. In addition, the participants may not be equally familiar with the medical terms and thus could have different perceptions on the outcomes, which would influence eliciting the preferences [12]. Hence, we used specific lay descriptions for each outcome, instead of the medical terms, which were constructed as a function of health loss-clinical features, treatment modalities, and functional consequences or prognosis-on which the respondents relied to express their preferences (Additional file 1). We tried to simulate a typical manifestation for each outcome while being fully aware that this simplifies reality. We consulted clinical and methods experts to evaluate the lay descriptions. We stated the descriptions using as little technical terms as possible and supplemented anonymous pictures that would help respondents easily select their preferred outcome.

We originally developed the questionnaire in English and then translated it into Tigrigna and German-the local languages in the respective study sites. We consulted bilingual speakers and experts familiar with preference studies to check for consistency and appropriateness of the lay descriptions. We piloted the questionnaires twice in both sites, first on 20 individuals and then on 10 others in the second round of the pilot that helped rephrase the vignettes of the outcomes, and simplify the medical terms. Members of the research and additionally recruited health personnel conducted the interviews. The recruits were trained, overseen, and assisted when the need arose.

The preference questions were designed using the object case Best-Worst Scaling (BWS) method [22], by which participants had to select a pair of best and worst outcomes at a time from presented scenarios. The BWS measures utility or preferences efficiently from fewer respondents. It overcomes the methodological and psychometric weakness of other methods, including visual analogue scale (VAS), pair comparisons, and time tradeoff [23, 24]. In our study, the terms 'best' and 'worst' refer to the most and least worrisome clinical outcome, respectively. We used the Balanced Incomplete Block Design (BIBD) to get efficiently designed choice sets (Additional file 1). This provided 13 scenarios, with four outcomes in each scenario, to be answered per respondent, where every outcome appeared in four different scenarios and coexisted with another one just once. This design yielded 2860 paired-responses and 34,320 impliedcomparisons in the pooled data.

Before they started the BWS procedure, respondents were asked to express their perceived severity using VAS for each of the 13 outcomes in order to familiarize them with the descriptions. This also offered a comparison of the preferences from the VAS with BWS.

Mekelle University, Ethiopia and the University of Zurich granted the study an expedited approval. The study did not involve any physical or laboratory examination, nor did it collect any respondents' identifiers, and thus we obtained oral consent from each participant after they were briefed about the study's purpose and procedure, and as well as the confidentiality of the anonymized data.

\section{Sample size}

There is no standard sample size estimation method for BWS studies [25]. We reviewed studies that used BWS and took the median sample size of 220 (this included $10 \%$ contingency for non-responses) from studies with similar number of choice sets $[22,26]$.

\section{Statistical analysis}

We used R (3.2.2) and STATA (13.0) for data analyses and SAS (9.4) to generate the BIBD. First, we used $\mathrm{R}$ algorithms to convert the respondents' dataset into structurally convenient data frame for preference analyses. We assigned 1 for an outcome selected as most worrisome, -1 for least worrisome, and 0 unless selected otherwise.

We estimated the preferences in log-transformed coefficients (log-odds) and probability terms using different analysis ways; thereafter, we refer to these as relative preferences, and preference weights, respectively. We ran conditional logit-regression models on the 34,320, 18,720 , and 15,600 paired-comparisons on the pooled and survey-specific data to get the relative preference values for the outcomes. Unlike standard analyses, this method modeled response as a function of differences in preferences, that indicates utility or preference relations [27] and took into account the correlation of responses within an individual and a scenario. The result from this method explains the relationship with microeconomic theory, which has implications for statistical inference [28]. The model yields preferences on log-scaled linear line on which the different outcomes take relative positions that imply their relative preferences or importance.

We also estimated preferences weights in probability terms; i.e., the probability of an outcome being selected as most worrisome given the rest comparators. First we calculated standardized mean of frequencies of outcome selected as 'best' minus 'worst' (B-W) scores and standard deviation for each pair of responses. We then ran multiple treatment comparison method (i.e., multivariate random-effects meta-analysis model) on the outcomes to obtain summary of standardized mean differences for the $\mathrm{B}-\mathrm{W}$ scores for each outcome. With flat priors and posterior normal distribution of the standardized mean 
difference of B-W scores for each pair of responses and variance equal to the frequentist estimates from the above results, we calculated the probability that an outcome is selected as the most worrisome, the second worrisome, and so on using Markov chain Monte Carlo method in the Bayesian model. This yielded the probability of being most worrisome for each outcome in every trial. We then estimated the surface under the cumulative ranking curve for each outcome in probability terms as shown in Fig. A of the Additional file 1. A preference weight of 1 means that the outcome is certain to be the most worrisome while 0 corresponds to the outcome to be the least worrisome. To estimate confidence intervals, we simulated normal random variates with means defined by the preference weights and variance by the between survey estimates. We then drew 1000 bootstrap samples and repeated the means for each sample. We took the 2.5th and 97.5th percentiles in the distribution about each preference weight.

We assessed measurement error and internal consistency within and between the surveys by portraying a heat map of response probability for the $13 \times 13$ paired-comparisons. We also ran linear regression to test if survey site and socio-demographic factors affected the preferences.

\section{Results}

\section{Characteristics of respondents}

We obtained responses from all the 220 participants. Table 1 shows socio-demographic and other characteristics of respondents by survey site. Respondents from Switzerland had a higher proportion of educational attainment and employment than those from Ethiopia. While age distribution was truncated by sampling eligibility at 40 years, the participants were older and the probability of morbidity was higher in Switzerland.

\section{Consistency of responses}

Figure 1 shows heat maps of probability for the $13 \times 13$ possible combinations of outcomes for the surveyspecific data. Each cell indicates the probability that respondents selected the first comparator in a pair as the most worrisome. The matrix of the probabilities is arranged from 0 to 1 , which corresponds to yellow and orange, respectively. The smooth transition from yellow at the right lower corner to orange at the left upper corner of the map indicated a small amount of measurement error and high internal consistency of the responses within and between the sites. In support to this, we included a question that asked respondents how difficult the descriptions and the BWS procedure were to understand. Eighty four percent (84/100) of the respondents in Ethiopia and 96\% (115/120) in Switzerland reported that they well understood the questions and the procedures of the study; where this slight difference corresponded to the slightly less consistency on the heat map from Ethiopia.

\section{Preferences}

The relative preference values (log-odds) of the outcomes compared to the treatment discontinuation along with 95\% Cl's are shown in Table 2. Severe stroke, severe myocardial infarction (MI), and cancer were the three most worrisome outcomes. The least worrisome outcomes were treatment discontinuation and nausea/headache. The orders of the relative preferences of all outcomes were consistent across the three models (corresponding to the surveys in Ethiopia, Switzerland and the pooled data) with precise confidence intervals. Since relative scales are not appropriate for comparison, we normalized the values and found that the preferences were similar between Ethiopia and Switzerland. On the relative scale, two point estimates showed reversal in order; i.e., in Ethiopia, heart failure was preferred to moderate MI, and acute kidney failure to unstable angina as compared to the Swiss data. However, the uncertainty intervals ruled out the possibility of flip of orders. The uncertainty intervals of the survey-specific data included the null value for nausea/headache, which suggest respondents had similar preferences for nausea/ headache and the treatment discontinuation. In the pooled data, the interval did not contain the null value probably due to the doubled sample size.

The preference weights in probability terms are presented in Table 2. In the models run on the pooled and separate surveys, the preference weights were patterned in a similar way as the orders in the relative scale, and with close magnitudes between Ethiopia and Switzerland. In the pooled data, severe stroke outranked all with preference of 1 followed by severe MI, 0.913 (95\% CI 0.889-0.942); cancer, 0.846 (0.829-0.855); moderate stroke, 0.735 (0.671-0.802); and moderate MI, 0 . 664 (0.611-0.715); whereas nausea/headache, 0.060 (0. 034-0.094); treatment discontinuation, 0.090 (0.023-0. $123)$; and myopathy, $0.230(0.228-0.238)$ were the least worrisome outcomes.

We checked the measurement agreement between methods, and the consistency of respective results between the survey sites. Figure 2 shows that the relative preferences, standardized B-W scores and preference weights were linearly related as depicted by the relational line and the corresponding increase in circle areas. Figure 3 presents observed preferences with a local smoothing over survey-specific versus pooled data using different preference scales. The results were consistent between the survey data with overlapping uncertainty intervals of estimates from the linear regression 
Table 1 Characteristics of participants involved in the preference eliciting study

\begin{tabular}{|c|c|c|c|}
\hline Characteristics & Pooled $(n=220)$ & Ethiopia $(n=100)$ & Switzerland $(n=120)$ \\
\hline \multicolumn{4}{|l|}{ Sex } \\
\hline Male, n(\%) & $111(50.4)$ & $54(54.0)$ & $57(47.5)$ \\
\hline Female & $109(49.6)$ & $46(46.0)$ & $63(52.5)$ \\
\hline \multicolumn{4}{|l|}{ Age } \\
\hline Mean (SD) & $52.9(0.6)$ & $49.7(0.7)$ & $55.6(0.8)$ \\
\hline $40-64$ & $195(88.6)$ & $96(96.0)$ & 99 (82.5) \\
\hline$\geq 65-81$ & $25(11.4)$ & $4(4.0)$ & $21(17.5)$ \\
\hline \multicolumn{4}{|l|}{ Education } \\
\hline Mean in years (SD) & $10.12(0.4)$ & $6.55(0.6)$ & $13.1(0.4)$ \\
\hline None & $25(11.4)$ & $25(25.0)$ & 0 \\
\hline Primary & $46(20.9)$ & $42(42.0)$ & $4(3.3)$ \\
\hline Secondary & $70(31.8)$ & $16(16.0)$ & $54(45.0)$ \\
\hline Higher & 79 (35.9) & $17(17.0)$ & $62(51.7)$ \\
\hline \multicolumn{4}{|l|}{ Job } \\
\hline Salaried & $107(48.2)$ & $35(35.0)$ & $71(59.2)$ \\
\hline Own business & $46(20.9)$ & $24(24.0)$ & $22(18.3)$ \\
\hline Pensioned & $28(12.7)$ & $4(4.0)$ & $24(20.0)$ \\
\hline No job & $39(18.2)$ & $37(37.0)$ & $3(2.5)$ \\
\hline Current or previous statin users & $20(9.1)$ & $8(8.0)$ & $12(10.0)$ \\
\hline \multicolumn{4}{|l|}{ Co-living person } \\
\hline Alone & $48(21.8)$ & $11(11.0)$ & 37 (30.8) \\
\hline Family & $171(78.2)$ & 89 (89.0) & $83(69.2)$ \\
\hline \multicolumn{4}{|l|}{$\begin{array}{l}\text { Respondents understand the } \\
\text { content and procedure of } \\
\text { the questionnaire }\end{array}$} \\
\hline Strongly agree & $83(37.7)$ & $7(7.0)$ & $76(63.4)$ \\
\hline Agree & $116(52.7)$ & 77 (77.0) & $39(32.5)$ \\
\hline Neither & $20(9.1)$ & $16(16.0)$ & $4(3.3)$ \\
\hline Disagree & $1(0.5)$ & 0 & $1(0.8)$ \\
\hline \multicolumn{4}{|l|}{ Morbidity } \\
\hline None & $149(67.7)$ & $81(81.0)$ & $68(56.7)$ \\
\hline Yes $^{a}$ & $71(32.3)$ & 19 (19.0) & $52(43.3)$ \\
\hline
\end{tabular}

${ }^{a}$ Hypertension, type 2 diabetes, join/muscle disease, cancer, psychiatric disease were most frequently reported

(Additional file 1) and high Lin's Concordance Correlation Coefficient between the pooled and survey-specific data, 0.98 (95\% CI 0.97 to 0.99 ) for the normalized logodds and 0.97 (95\% CI 0.90-0.99) for both preference weights and standardized B-W scale. Plot 'd' presents poor relationship of VAS versus preference weights. The Concordance Correlation Coefficient between these measures was 0.68 (95\% CI $0.40-0.85)$ that indicated VAS was a weak method to quantify preference values.

Reasons most frequently mentioned for influencing respondents' preference were prognosis, 28\% (126 out of 450 responses); severity, 27\% (122/450); and curability, $21 \%(95 / 450)$ of the clinical outcomes. The linear regression did not find factors with consistent pattern of influence on the preferences (Additional file 1).

\section{Discussion}

\section{Summary of results}

We found in this study that preferences of respondents from Ethiopia and Switzerland were similar despite large differences in the socio-cultural, health system, and economic contexts. Severe stroke, severe MI and cancers were ranked the worst outcomes while myopathy, nausea/headache, and treatment discontinuation were perceived as the least worrisome outcomes. 


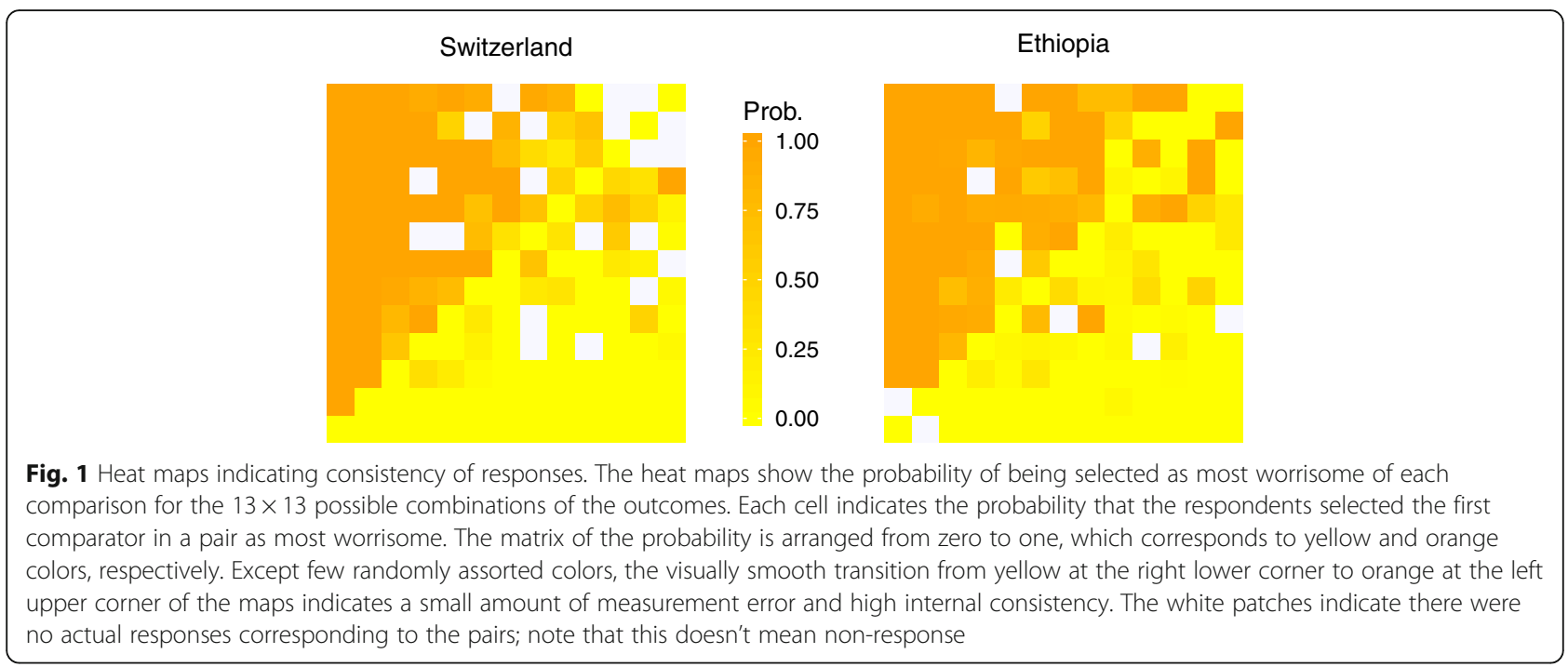

\section{Interpreting and discussing the findings in comparison with the literature}

Although the universality of preference weights across culturally, and socio-economically diverse environments is much discussed in the literature [15], our study found consistent preferences for the outcomes between the study sites taken from the Sub-Saharan African and European regions. Indeed, the GBD study that involved wide ranges of clinical outcomes did not show substantially variation of preferences across social contexts [15]. The hypothesis of preference weight variations across settings may stem from previous approaches that had used welfare loss to define the weights instead of health loss $[29,30]$. Quantifying preferences with an aim to measure welfare loss is likely to be affected by a society's level of welfare such as wealth and availability of health insurance. On that account, we predicated that cost or affordability, which greatly varies across the study areas, should not be regarded as a decisive outcome to influence statin prescribing decision because this could bias preferences of people from less affluent regions [31, 32]. Clinical guidelines emphasize on health loss, not welfare loss. Cost of treatment should be managed in a different way once it is established that net benefit of statins outweighs their harms. We controlled potential influences that may contribute to response variation within and across societies by specifying clear constructs of the clinical outcomes in order to ease, standardize, and decrease ambiguity of preference measurements among the participants with varying individual characteristics, such as educational level and awareness. This might have helped attenuate possible inter-personal or cross-environmental preference discrepancies.

We expected a wide range of preference magnitudes between the outcomes and, indeed, we found greatly distinct preference weights in our study ranging from 1 for severe stroke and 0.060 for nausea and headache in the pooled data. The preferences of the other outcomes spread out in between. In the context of prescribing decision, this suggests that severe stroke is the most worrisome and that patients may opt to take statins to prevent risk of severe stroke at the expense of possible unwanted effects. At policy level, these greatly differing values imply that clinical guidelines need to consider the preference values to quantitatively measure the benefitharm balance of outcomes related to statins. We also presented alternative measures in term of a relative scale to be used in similar way for gradating the importance of the outcomes. For instance, severe stroke was about six times more important than nausea/headache or treatment discontinuation. However, relative scales should not be used to compare the preferences between samples or varying settings, unless they are normalized.

We compared our results with the disability weights published by the European and GBD studies although estimates from these studies are less likely to apply to specific clinical decision-making contexts [15]. The weight for severe stroke, for example, was 0.539 in the GBD study, which significantly differs from the weight we obtained (i.e., 1.00). These studies estimated disability weights for a wide array of diseases and injuries with the aim of measuring DALYs [15]. They compared a given disease with another random disease with high chance of that disease being paired with another more severe one. Consequently, some moderate diseases could get higher weights if randomly paired with a milder, or lower if paired with a severe one, which would to a spurious conclusion. It was not also possible in the GBD study to compare all diseases relative to one another and that the method used could not handle the comparisons in an efficient way [33]. Furthermore, such weights could not be used for all clinical decision-contexts because 


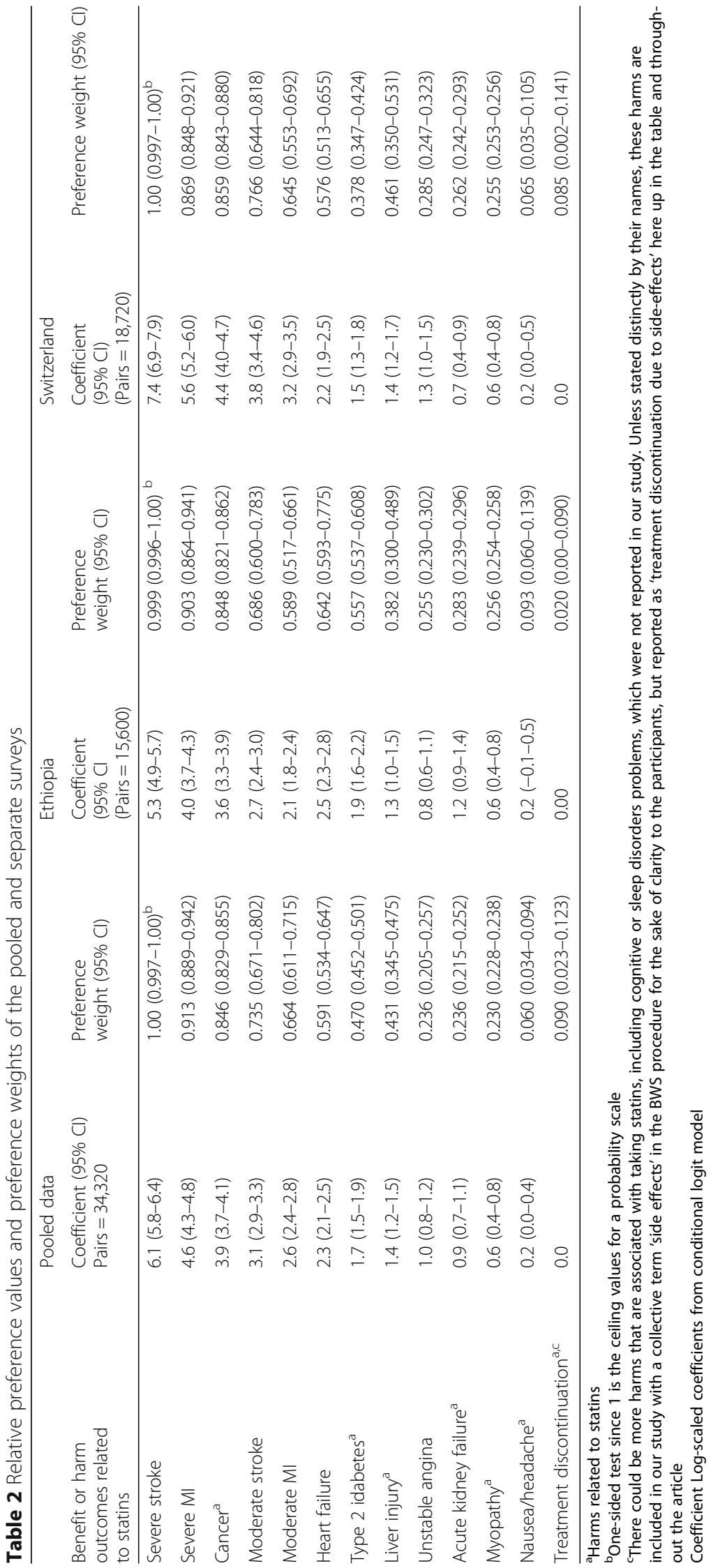




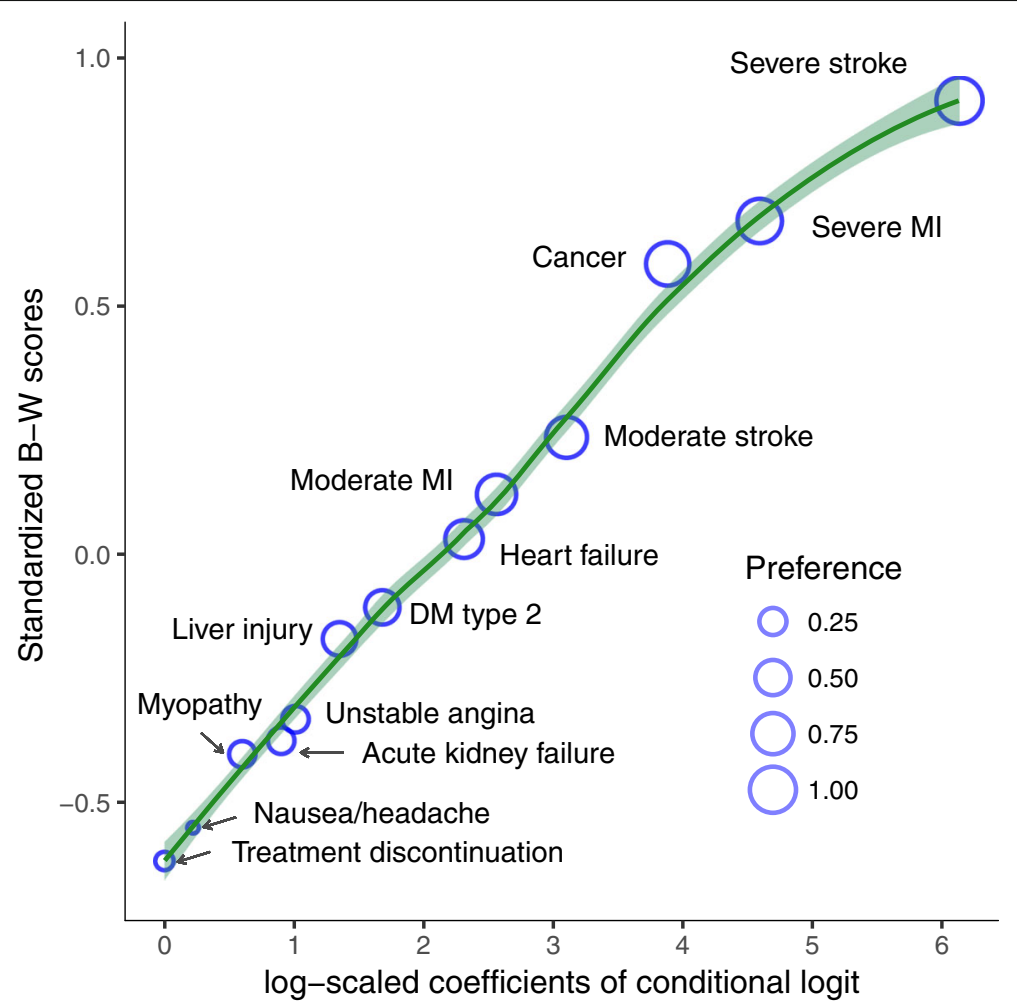

Fig. 2 Relationships of preference measurement methods. The smoothed line and increase in circle size portray the relationship between the preference measures

they could be different if a different decision context is considered, like the statin prescribing scenario, in which preferences are influenced by the number, clinical feature and prognosis of the considered outcomes. For example, there are only about a dozen statin-associated benefit and harm outcomes on which individuals depend to make preference tradeoffs. As a result, the expected preference weights of these outcomes simply cannot be similar with those calculated for other purposes and decision contexts, such as for measuring DALY in the GBD study where many outcomes were considered.

\section{Implications of findings}

The preference weights calculated in our study have important implications for guideline developers and clinicians. No empirical evidence about preferences is available for the statin-associated benefit and harm outcomes and thus clinical guideline developers did not consider when framing the clinical guideline of statins for CVD prevention. There is also a lack of quantitative evaluations of the benefit-harm balance of statins that would needs to include patient-important outcomes, the effects of statins on these outcomes, baseline incidences of the population of interest to calculate absolute effects as well as preference weights for different outcomes [1, 34]. Ignoring preferences and baselines risks could lead to over treatment, especially in primary population where the risk of CVD is low and uncertain. Our study provides preferences values of the outcomes that are much needed for quantitatively assessing the benefit and harm balance of statins. The greatly differing preference values among the outcomes may also give clinicians some guidance for considering patients' preferences when prescribing statins, and as well as baseline evidence to researchers to develop personalized benefitharm assessment models.

\section{Strengths and limitations of the study}

Our study has a number of strengths. We clearly defined our decision-context and constructed the descriptions of the clinical outcomes and the answering options in an iterative way to make sure respondents understand them and give us valid preferences. Also, we employed faceto-face interviews to enhance data quality and minimize non-response rate. We pursued efficient methods for comparing all outcomes relative to each other from fewer respondents and analyzed the data with robust methods. Another key strength was testing the hypothesis on possible variation in the preference weights across divergent environments, which is critical in deciding whether setting-specific prescribing practices are needed. 

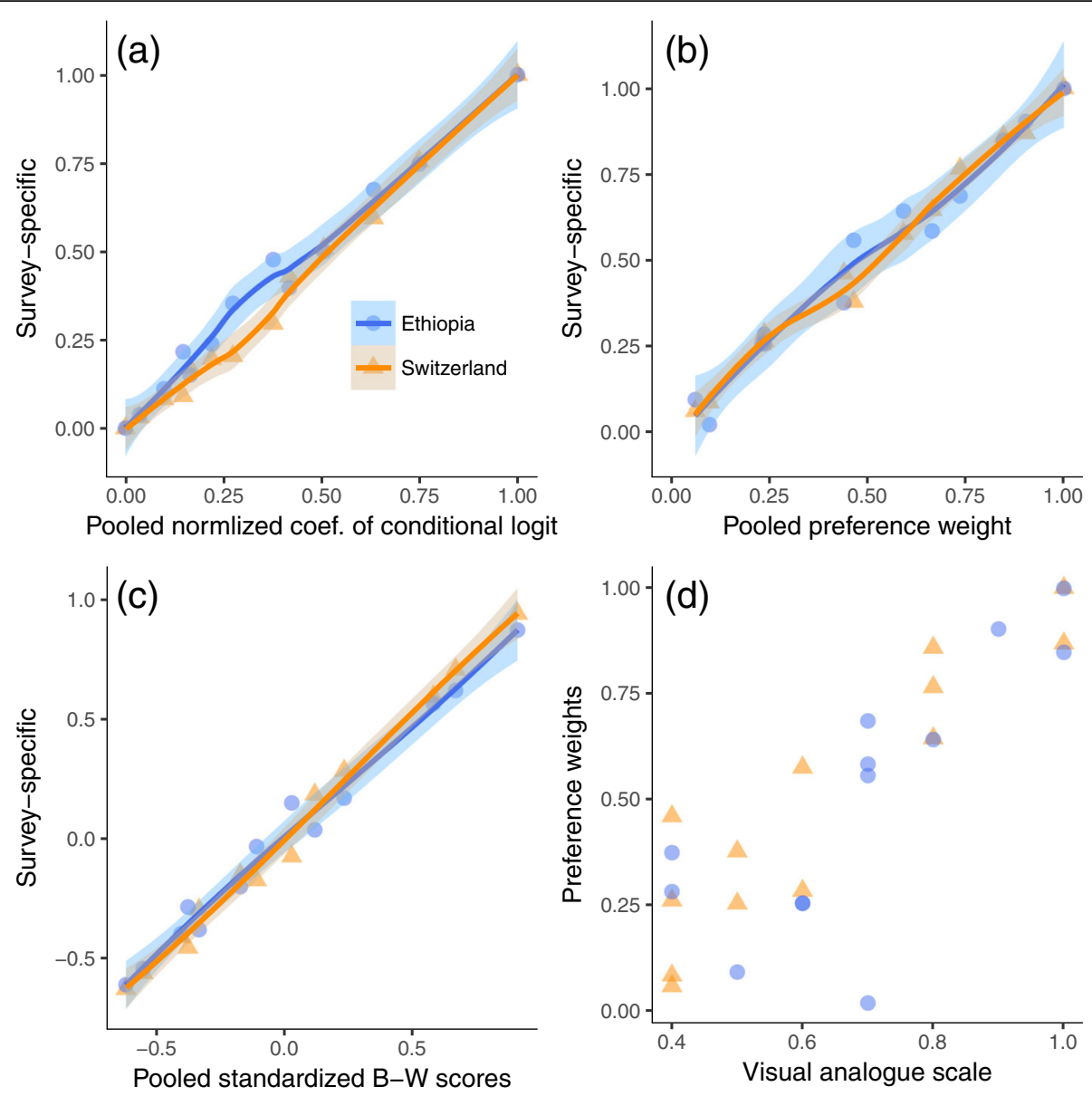

Fig. 3 Measurement agreement and consistency of results between the pooled data and specific surveys. Plots (a), (b) and (c) show observed and smoothed uncertainty interval of measurements between surveys. Plot $\mathbf{d}$ shows correlation between VAS and preference weight. The dots represent the benefit and harm outcomes specified in Fig. 2

Our study might also have certain limitations regarding the preference estimates. The study sites were not randomly selected. Although we tried to design the tool in a way people would give their preference responses emphasising on the lay descriptions regardless of differences in socio-demographic backgrounds, our data could not rule out the possibility of getting different estimates if we had included other sites. Besides since the descriptions were as short as possible, respondents might give responses based on own perceptions out of the scope of the specified lay descriptions. Generally, there was high internal consistency and low measurement errors, except a few erratic paired-responses from both sites, which may show that a few respondents were skeptical or gave responses based on their perception independent of the lay descriptions.

\section{Conclusions}

Our study provides much needed evidence on preferences related to statins for primary CVD prevention that clinical guideline developers could take into account when developing recommendations. Contrary to popular opinion, our empirical data show that preferences were similar across environments that greatly differ in terms of demographic, socio-cultural and economical perspectives. However, we recommend inclusion of more countries in future studies to make robust conclusion about generalizability of the preferences to more environments.

\section{Additional file}

Additional file 1: This contains an example lay description for chest pain, BIBD design of outcomes, surface under the cumulative ranking curves of benefit and harm outcomes as measure of the preferences, and results of linear regression assessing influence of participants' characteristics on preference values. (PDF 376 kb)

\section{Abbreviations}

BIBD: Balanced Incomplete Block Design; B-W: Best minus Worst scores; BWS: Best-worst Scaling; CVD: Cardiovascular Disease; DALY: DisabilityAdjusted Life Years; GBD: Global Burden of Disease; RCT: Randomized Controlled Trials; VAS: Visual Analogue Scale 


\section{Acknowledgments}

We thank all patients involved in piloting the questionnaire, study participants and interviewers in Ethiopia and Switzerland, and clinical staff in the travel clinic of the University of Zurich. We acknowledge the Swiss Federal Government and North-South cooperation at the University of Zurich for funding the study.

\section{Funding}

The North-South Cooperation at the University of Zurich (F-42320-71-01) and Swiss Federal Government (2015.0858) funded the study. The funders had no role in the study design, data collection, data analysis, data interpretation, or writing of the report. All authors had full access to the data in the study and the MAP had final responsibility for the decision to submit for publication.

\section{Availability of data and materials}

The datasets used and analyzed during the current study are available from the corresponding author on a reasonable request.

\section{Authors' contributions}

HGY designed the study protocol and questionnaire, implemented and supervised the study, analyzed the data, and drafted the first and final versions of the report. HEA contributed to the design of the questionnaire, checked data quality, and revised the drafts of the report. TY contributed to the study protocol and questionnaire, and revised the drafts of the report. MAP conceived the study; contributed to the study protocol and design of the questionnaire, implemented and supervised the study, contributed to the analysis, and made revisions on the report. All authors approved the final version. MAP is the guarantor of the study.

\section{Ethics approval and consent to participate}

The ethical boards of Mekelle University, Ethiopia (Ref.no ERC 0799/2016) and the University of Zurich (Req-2016-00414) granted our study an expedited approval. Oral consent was obtained from each participant after they were briefed about the study's purpose and procedure, and as well as the confidentiality of data.

\section{Competing interests}

The authors declare that they have no competing interests

\section{Publisher's Note}

Springer Nature remains neutral with regard to jurisdictional claims in published maps and institutional affiliations.

\section{Author details}

'Department of Epidemiology, Biostatistics \& Prevention Institute, University of Zurich, Hirschengraben 84, CH-8001 Zurich, Switzerland. ${ }^{2}$ School of Public Health, College of Health Sciences, Mekelle University, Ayder, Mekelle, Ethiopia. ${ }^{3}$ Department of Public Health, China Medical University, Taichung, Taiwan.

Received: 29 May 2017 Accepted: 10 May 2018

Published online: 18 May 2018

\section{References}

1. Bibbins-Domingo K, Grossman DC, Curry SJ, Davidson KW, Epling JW, García $F A R$, et al. Statin use for the primary prevention of cardiovascular disease in adults. JAMA. 2016;316:1997-2007.

2. Mortensen MB, Nordestgaard BG. Comparison of five major guidelines for statin use in primary prevention in a contemporary general population. Ann Intern Med. 2018;1682:85.

3. Pagidipati NJ, Navar AM, Mulder H, Sniderman AD, Peterson ED, Pencina MJ. Comparison of recommended eligibility for primary prevention statin therapy based on the US preventive services task force recommendations vs the ACC/AHA guidelines. JAMA. 2017;31715:1563-7.

4. Wang H, Naghavi M, Allen C, Barber RM, Bhutta ZA, Carter A, et al. Global, regional, and national life expectancy, all-cause mortality, and cause-specific mortality for 249 causes of death, 1980-2015: a systematic analysis for the global burden of disease study 2015. Lancet. 2016;388:1459-544.
5. Daar A, Singer P, Leah Persad D, Pramming S, Matthews D, Beaglehole R, et al. Grand challenges in chronic non-communicable diseases. Nature. 2007; 450:494-6.

6. Ong HT. Evidence-based prescribing of statins: a developing world perspective. PLoS Med. 2006;33:303-7.

7. Otto CM. Statins for primary prevention of cardiovascular patients need better tools to navigate divergent recommendations. BMJ. 2016;355:1-2.

8. Naci H, Brugts J, Ades T. Comparative tolerability and harms of individual statins : a study-level network meta-analysis of 246955 participants from 135 randomized, controlled trials. Circ Cardiovasc Qual Outcomes. 2013;64:390-9.

9. Chou R, Dana T, Blazina I, Daeges M, Jeanne TL. Statins for prevention of cardiovascular disease in adults. JAMA. 2016;316:2008-24.

10. Anderson TJ, Jean G, Pearson GJ, Barry AR, Couture P, Dawes M, et al. 2016 Canadian cardiovascular society guidelines for the management of dyslipidemia for the prevention of cardiovascular disease in the adult. Can J Cardiol. 2016;32:1263-82

11. Gail MH, Costantino JP, Bryant J, Croyle R, Freedman L, Helzlsouer K, et al. Weighing the risks and benefits of tamoxifen treatment for preventing breast cancer. J Natl Cancer Inst. 1999:91:1829-46.

12. Montori VM, Brito JP, Murad MH. The optimal practice of evidence-based medicine incorporating patient preferences in practice quidelines. JAMA. 2013;310:2503-4.

13. Puhan MA, Yu T, Stegeman I, Varadhan R, Singh S, Boyd CM. Benefit-harm analysis and charts for individualized and preference-sensitive prevention: example of low dose aspirin for primary prevention of cardiovascular disease and cancer. BMC Med. 2015;13:1-11.

14. Puhan MA, Singh S, Weiss CO, Varadhan R, Boyd CMA. Framework for organizing and selecting quantitative approaches for benefit-harm assessment. BMC Med Res Methodol. 2012;12:1-12.

15. GBD Disability Weights Measurement Collaborators. Common values in assessing health outcomes from disease and injury: disability weights measurement study for the global burden of disease study 2010. Lancet. 2012;380:2129-43.

16. Haagsma JA, Maertens de Noordhout C, Polinder S, Vos T, Havelaar AH, Cassini $A$, et al. Assessing disability weights based on the responses of 30,660 people from four European countries. Popul Health Metr. 2015;13:10.

17. Baltussen RMPM, Sanon M, Sommerfeld J, Würthwein R. Obtaining disability weights in rural Burkina Faso using a culturally adapted visual analogue scale. Health Econ. 2002;11:155-63.

18. Bühler $S$, Rüegg R, Steffen R, Hatz C. A profile of travelers-an analysis from a large Swiss travel clinic. J Travel Med. 2014;21:324-31.

19. Brugts JJ, Yetgin T, Hoeks SE, Gotto a M, Shepherd J, Westendorp R, et al. The benefits of statins in people without established cardiovascular disease but with cardiovascular risk factors: meta-analysis of randomised controlled trials. BMJ. 2009;338:b2376

20. Naci H, Brugts JJ, Fleurence $\mathrm{R}$, Tsoi B, Toor H, Ades AE. Comparative benefits of statins in the primary and secondary prevention of major coronary events and all-cause mortality : a network meta-analysis of placebocontrolled and active-comparator trials. Eur J Prev Cardiol. 2013;20:1-17.

21. Rosenson RS, Baker SK, Frcp C, Jacobson TA, Kopecky SL, Parker BA. An assessment by the statin muscle safety task force : 2014 update. J Clin Lipidol. 2014:8:S58-71.

22. Mühlbacher AC, Kaczynski A, Zweifel P, Johnson FR. Experimental measurement of preferences in health and healthcare using best-worst scaling : an overview. Health Econ Rev. 2016;62:1-14

23. Arnesen T, Nord E. The value of DALY life: problems with ethics and validity of disability adjusted life years. Br Med J. 1999;319:1423-5.

24. Krabbe PFM, Essink-Bot ML, Bonsel GJ. The comparability and reliability of five health-state valuation methods. Soc Sci Med. 1997:45:1641-52.

25. Yang JC, Johnson FR, Kilambi V, Mohamed AF. Sample size and utilitydifference precision in discrete-choice experiments: a meta-simulation approach. J Choice Model. 2015;16:50-7.

26. Bridges JF, Evers SMAA, Hiligsmann M. Using best-worst scaling to investigate preferences in health care. PharmacoEconomics. 2016;34:1195-209.

27. Louviere JJ, Wasi N, Street D, Islam T, Marley AAJ. Modeling the choices of individual decision-makers by combining efficient choice experiment designs with extra preference information. J Choice Model. 2008;11:128-64.

28. Mühlbacher AC, Zweifel P, Kaczynski A, Johnson FR. Experimental measurement of preferences in health care using best-worst scaling (BWS): theoretical and statistical issues. Health Econ Rev. 2015;6:5. 
29. Health HDM. Well-being, and measuring the burden of disease. Popul Health Metr. 2012;10:1-7.

30. Hausman D. Valuing health: a new proposal. Health Econ. 2010;19:280-96.

31. Kankeu H, Saksena P, Xu K, Evans DB. The financial burden from noncommunicable diseases in low- and middle-income countries: a literature review. Heal res policy Syst 2013;11:1-12.

32. Hosseinpoor AR, Bergen N, Mendis S, Harper S, Verdes E, Kunst A, et al. Socioeconomic inequality in the prevalence of noncommunicable diseases in low- and middle-income countries: results from the world health survey. BMC Public Health. 2012;12:474.

33. Haagsma JA, van Beeck EF, Polinder S, Hoeymans N, Mulder S, Bonsel GJ. Novel empirical disability weights to assess the burden of non-fatal injury. Inj Prev. 2008;14:5-10.

34. Yu T, Vollenweider D, Varadhan R, Li T, Boyd C, Puhan MA. Support of personalized medicine through risk-stratified treatment recommendationsan environmental scan of clinical practice guidelines. BMC Med. 2013;11:7.

Ready to submit your research? Choose BMC and benefit from:

- fast, convenient online submission

- thorough peer review by experienced researchers in your field

- rapid publication on acceptance

- support for research data, including large and complex data types

- gold Open Access which fosters wider collaboration and increased citations

- maximum visibility for your research: over $100 \mathrm{M}$ website views per year

At BMC, research is always in progress.

Learn more biomedcentral.com/submissions 